\title{
Experimental Investigation of the Nuclear Structure in the Neutron-Rich ${ }^{180} \mathrm{Hf}$
}

\author{
P. Vasileiou ${ }^{1}$, T.J. Mertzimekis ${ }^{1}$, A. Chalil ${ }^{1,2}$, A. Zyriliou $^{1}$, \\ S. Pelonis ${ }^{1}$, M. Efstathiou ${ }^{1}$, V. Lagaki ${ }^{1}$, G. Siltzovalis ${ }^{1}$, \\ P. Koseoglou ${ }^{3}$, D. Bonatsos ${ }^{4}$, A. Martinou ${ }^{4}$, N. Minkov ${ }^{5}$, \\ N. Mărginean ${ }^{6}$, C. Mihai ${ }^{6}$, N. Florea ${ }^{6}$, S. Ujeniuc ${ }^{6}$, \\ A. Turturica ${ }^{6}$, C. Costache ${ }^{6}$, R. Mihai ${ }^{6}$, L. Stan ${ }^{6}$, D. Filipescu ${ }^{6}$, \\ S. Toma ${ }^{6}$, I. Gheorghe ${ }^{6}$, I. Dinescu ${ }^{6}$, A. Ionescu ${ }^{6}$, L. Stoica ${ }^{6}$, \\ S. Calinescu ${ }^{6}$, A. Oprea ${ }^{6}$, A. Stoica ${ }^{6}$, C. Sotty ${ }^{6}$, C. Clisu $^{6}$, \\ C. $\mathrm{Nita}^{6}$, C. $\mathrm{Neascu}^{6}$
}

${ }^{1}$ National \& Kapodistrian University of Athens, Zografou Campus, GR-15784, Athens, Greece

${ }^{2}$ IRFU, CEA, Université Paris-Saclay, FR-91190, Paris, France

${ }^{3}$ Institut für Kernphysik, TU Darmstadt, Schlossgartenstr. 9, D-64289, Darmstadt, Germany

${ }^{4}$ Institute of Nuclear and Particle Physics, NCSR "Demokritos", GR-15310, Aghia Paraskevi, Greece

${ }^{5}$ Institute of Nuclear Research and Nuclear Energy, Bulgarian Academy of Sciences, BG-1784, Sofia, Bulgaria

${ }^{6}$ Horia Hulubei National Institute of Physics and Nuclear EngineeringIFIN-HH, R-077125, Bucharest, Romania

Received 25 October 2021

doi: https://doi.org/10.55318/bgjp.2021.48.5-6.618

\begin{abstract}
The structure of ${ }^{180} \mathrm{Hf}(Z=72)$ is of particular interest, as the nucleus features a rotational ground state band, but also several other collective bands, $\mathrm{K}$-isomers, and non-band members, which are generally poorly known. This work features preliminary results from a recent experimental campaign at IFIN-HH, Romania, aimed at measuring lifetimes of excited states in the neutron-rich ${ }^{180} \mathrm{Hf}$, by means of the RDDS technique. The ${ }^{181} \mathrm{Ta}\left({ }^{11} \mathrm{~B},{ }^{12} \mathrm{C}\right){ }^{180} \mathrm{Hf}$ proton pick-up reaction was used to populate excited states in the ${ }^{180} \mathrm{Hf}$ nucleus. The $\gamma$ transitions depopulating these levels were detected using the ROSPHERE array, in its 25 HPGe configuration. The array was coupled to the SORCERER particle detector and a plunger device enabling the study of $\mathrm{p}-\gamma$ and $p-\gamma-\gamma$ coincident events. Six plunger distances were chosen, allowing for the construction of the decay curves of the observed $\gamma$ transitions, from which the lifetimes of the levels of interest can subsequently be deduced.
\end{abstract}

KEY WORDS: ${ }^{180} \mathrm{Hf}$, lifetime measurements, RDDS. 


\section{Experimental Investigation of ${ }^{180} \mathrm{Hf}$}

\section{Introduction}

Neutron-rich even-even nuclei located in the rare earth region of the nuclear chart present attractive cases for the study of phenomena related to the nuclear structure. Nuclei in this region are characterized by low-lying isomeric states [1, 2], shape coexistence [3] and significant deformations that can be attributed to the shape of the nuclear potential, new prevailing symmetries and, often, the emergence of octupole degrees of freedom [4-9].

The even-even Hf isotopic chain belongs in this category, and has been the subject of numerous theoretical studies (e.g. Refs. [10-14]). The multi-faceted structure of ${ }^{180} \mathrm{Hf}(Z=72)$ is of particular interest as the nucleus features a rotational ground state band (gsb), but also several other collective bands, Kisomers and non-band members, which are generally poorly investigated with regard to the lifetimes of the excited states, the transition rates, and the nuclear electromagnetic moments $[2,15]$.

The presence of a negative parity band built on top of a $\left(4^{-}\right)$isomeric state (Figure 1, band 2) and a band built on a $0_{2}^{+}(1109.1 \mathrm{keV})$, which suggests the occurrence of shape coexistence, are attractive cases to be investigated experimentally, collecting data that are currently unavailable. The recently developed proxy-SU(3) $[16,17]$ model predicts shape coexistence for the hafnium isotopes, with ${ }^{180} \mathrm{Hf}$ being well inside the shape coexistence window [17], which seeks experimental confirmation.

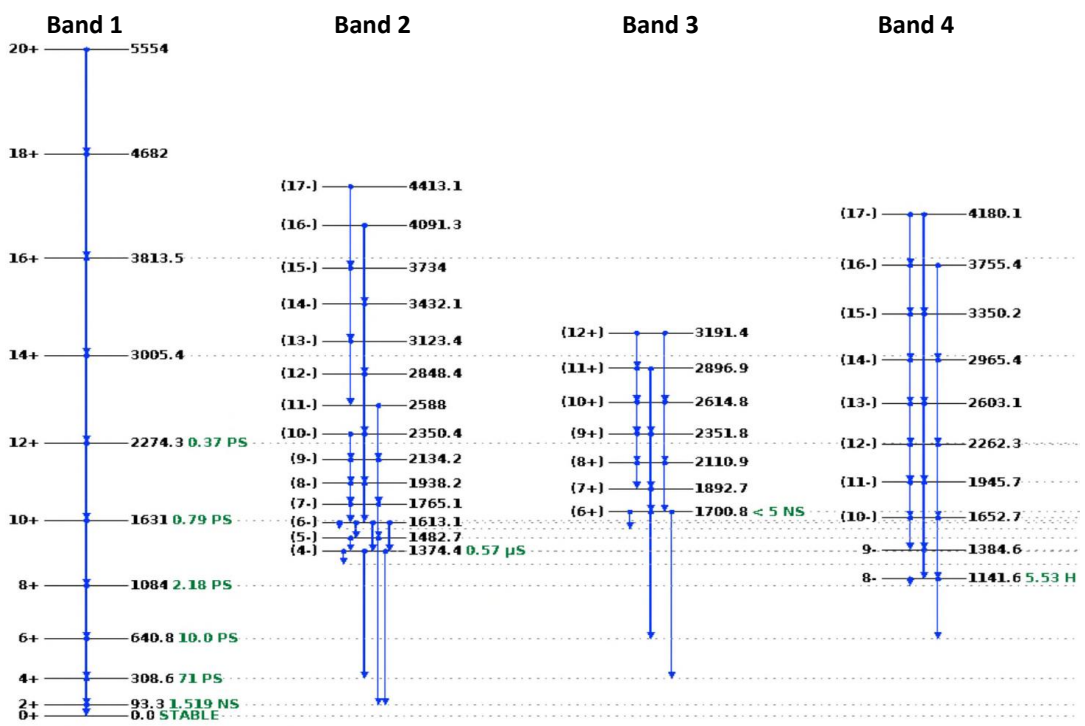

Figure 1. (Color online) Partial level scheme of ${ }^{180} \mathrm{Hf}$, adapted from Ref. [2]. 
It was only recently that the g.s. band lifetimes have been measured for this nucleus up to the $6_{1}^{+}$level, by means of fast-electronic scintillation timing [18]. An exploratory experiment was performed by our group, at IFIN-HH, in Romania, in April 2019, focused at the determination of spins, parities and mixing ratios of exited states in ${ }^{180} \mathrm{Hf}[19]$.

This work reports on the preliminary results of our most recent experimental campaign at the IFIN-HH laboratory, aimed at measuring lifetimes in the neutron-rich ${ }^{180} \mathrm{Hf}$, by means of the Recoil Distance Doppler Shift (RDDS) technique.

\section{Experimental Details}

\subsection{Experimental Setup}

The proton-pickup reaction ${ }^{181} \mathrm{Ta}\left({ }^{11} \mathrm{~B},{ }^{12} \mathrm{C}\right){ }^{180} \mathrm{Hf}$ was used to populate excited states in ${ }^{180} \mathrm{Hf}$ at the IFIN-HH laboratory, in Magurele, Romania. The ${ }^{11} \mathrm{~B}$ beam was accelerated at a laboratory energy of $47 \mathrm{MeV}$ by the 9 MV TANDEM accelerator, before bombarding a $1 \mathrm{mg} \cdot \mathrm{cm}^{-2}$ self-supported metallic Ta target (with a natural abundance of $99.99 \%$ in ${ }^{181} \mathrm{Ta}$ ) [2].

The $\gamma$-rays emitted during the decays of the products were detected with the use of the ROSPHERE [20] array, in its 25 HPGe configuration, providing a $4 \pi$ solid angle coverage. The SORCERER [21] solar cell particle detector device was coupled to the array, improving the ability to isolate events of interest through $\mathrm{p}-\gamma$ and $\mathrm{p}-\gamma-\gamma$ techniques. The detector array used in our experiments is illustrated in Figure 2.

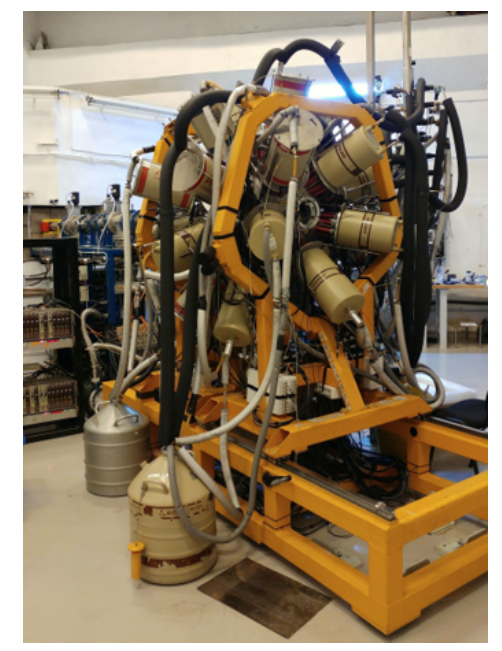

Figure 2. (Color online) The ROSPHERE detector array used in our experiment. 


\section{Experimental Investigation of ${ }^{180} \mathrm{Hf}$}

The Recoil Distance Doppler Shift (RDDS) technique [22] was apllied to measure lifetimes of excited states in ${ }^{180} \mathrm{Hf}$. Six different target-stopper distances were chosen, ranging from $15 \mu \mathrm{m}$ to $260 \mu \mathrm{m}$. The basics of the RDDS technique are briefly explained below.

\subsection{The Recoil Distance Doppler-Shift (RDDS) Method}

The Recoil Distance Doppler Shift (RDDS) is a well established method for picosecond lifetime measurements of excited states in nuclei [22]. At time $t=0$ a beam-induced nuclear reaction on a thin target produces the excited nuclear level of interest. Due to momentum transfer, the excited nucleus leaves the target with a velocity $v$, and is stopped inside the stopper foil, after a well-defined flight time $t_{f}=x / v$, where $x$ is the target-stopper separation distance. Depending on the distance, some nuclei cannot reach the stopper foil before decaying, and thus, they decay in flight. The corresponding $\gamma$ rays are Doppler shifted. As the target-stopper distance changes, the number of Doppler-shifted events change accordingly. From the comparison of the areas of the shifted and unshifted peaks, the lifetime can be measured.

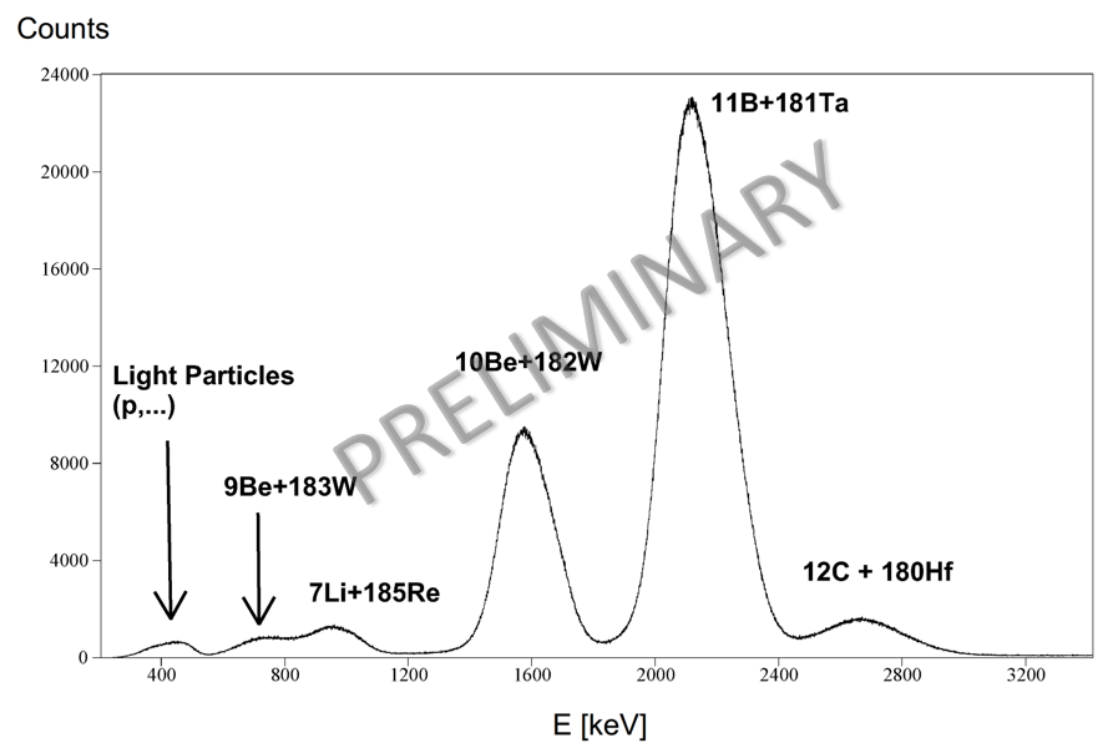

Figure 3. Typical particle spectrum recorded by the SORCERER array. The peaks correspond to different ejectiles detected, produced by the various reaction channels marked above their corresponding peaks. 


\section{Analysis and Preliminary Results}

By taking advantage of the SORCERER particle detector array, we were able to distinguish the various light ejectiles detected $\left({ }^{12} \mathrm{C},{ }^{11} \mathrm{~B}\right.$ etc $)$, thus identifying the products of the reaction $\left({ }^{180} \mathrm{Hf},{ }^{181} \mathrm{Ta}\right.$, etc $)$. A typical particle spectrum is shown in Figure 3.

Starting from the shortest distance $(15 \mu \mathrm{m})$, a gate was placed on the ${ }^{12} \mathrm{C}$ peak in the particle spectrum, corresponding to the ${ }^{180} \mathrm{Hf}$ gamma spectrum. Next, two-fold (i.e. $\gamma-\gamma$ ) events were sorted into three-dimensional cubes for further analysis, with the relative angle of each detector ring as the index number. Presented in Figure 4 are the spectra obtained for the different target-stopper distances, where the shifted and unshifted components of the $332 \mathrm{keV} 6_{1}^{+} \rightarrow 4_{1}^{+}$ transition in ${ }^{180} \mathrm{Hf}$ are shown clearly separated. This transition, albeit having a known lifetime of $\tau_{1 / 2}=10.0(7)$ ps in literature, can serve as a validation of our experimental method (plunger offsets, systematic errors etc).
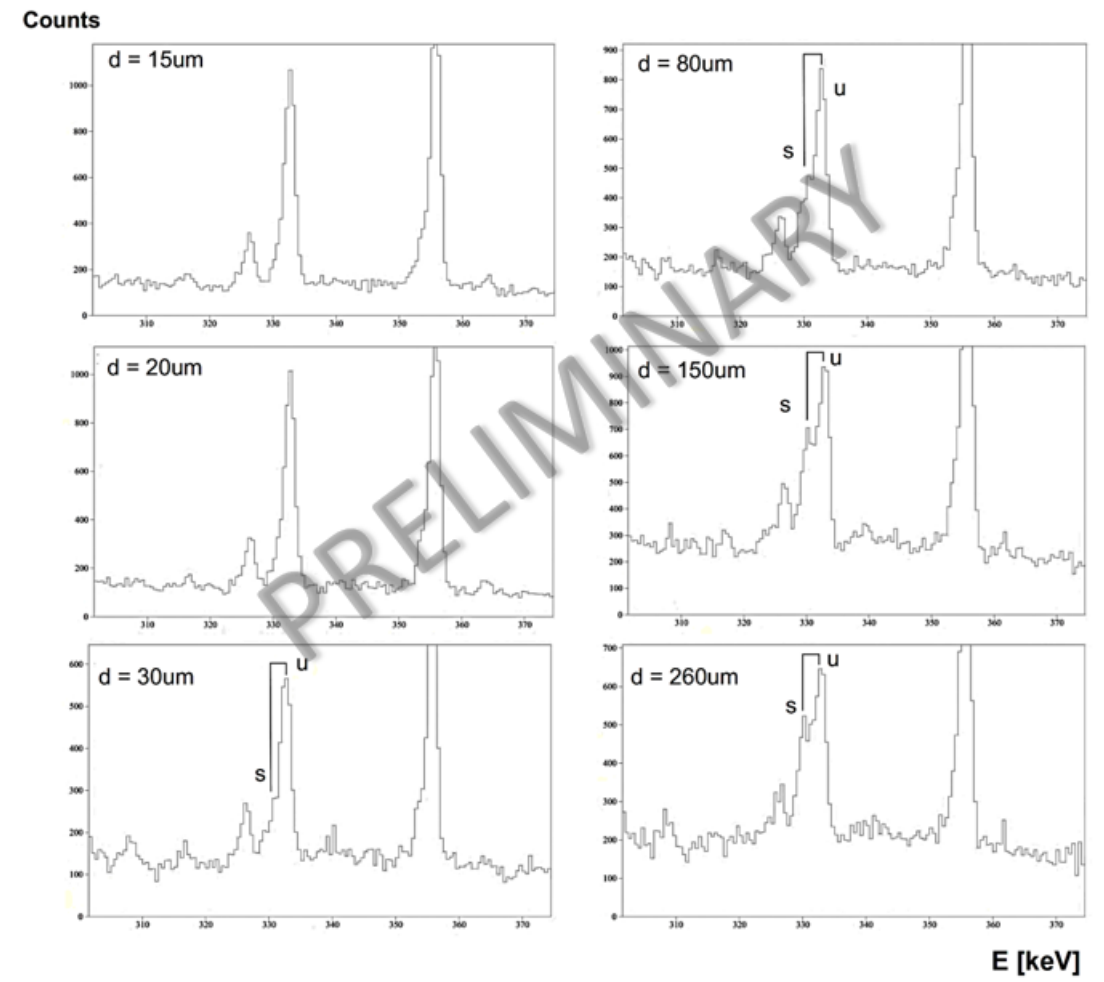

Figure 4. The $6+{ }_{1} \rightarrow 4_{1}^{+}$g.s. transition in ${ }^{180} \mathrm{Hf}$, measured for six different targetstopper distances $(15-260 \mu \mathrm{m})$. Notice the separation between shifted (s) and unshifted (u) components. This transition has a known lifetime of 10.0(7) ps [2] and can serve as a validation tool for the experimental method. 


\section{Experimental Investigation of ${ }^{180} \mathrm{Hf}$}

\section{Summary and Outlook}

Presented in this work are some preliminary results from the recent experimental campaign at IFIN-HH, in Romania, aimed at measuring lifetimes of excited states in the neutron-rich ${ }^{180} \mathrm{Hf}$. As it can be seen in these results, the experiment has produced various reaction channels, including the one populating the nucleus of interest, ${ }^{180} \mathrm{Hf}$.

As the analysis progresses, the rest of the observed transitions in ${ }^{180} \mathrm{Hf}$ will be thoroughly examined for shifts, and their lifetimes will be determined through their respective decay curves. Apart from ${ }^{180} \mathrm{Hf}$, other isotopes populated in this experiment (e.g. ${ }^{182} \mathrm{~W}, \mathrm{Pt}$ isotopes) will also be studied in an attempt to measure currently unknown lifetimes for the observed exited states.

In addition to experimental result, theoretical predictions stemming from a wide range of theoretical models, among which the recently developed proxy-SU(3), are expected to provide insight on the dynamical shape evolution in the studied isotopes, including shape coexistence which is predicted to exist in this region of the nuclear chart.

\section{Acknowledgements}

This research work was supported by the Hellenic Foundation for Research and Innovation (HFRI) under the HFRI PhD Fellowship grant (Fellowship Number: 101742/2019) for AZ. We acknowledge partial financial support by the Bulgarian National Science Fund (BNSF) under Contract No. KP-06-N28/6.

\section{References}

[1] P.M. Walker and G.D. Dracoulis (2001) Hyperfine Interact. 13583.

[2] Nudat2, National Nuclear Data Center, www.nndc.bnl.gov/nudat2/.

[3] V.K.B. Kota (1979) Phys. Rev. C 19521.

[4] L.M. Robledo et al. (2009) J. Phys. G 36115104.

[5] G.S. Simpson et al. (2009) Phys. Rev. C 80024304.

[6] K. Heyde and J.L. Woods (2011) Rev. Mod. Phys. 831467.

[7] K. Nomura et al. (2011) Phys. Rev. C 84054316.

[8] D. Bonatsos (2017) Eur. Phys. J. A 53148.

[9] D. Bonatsos et al. (2017) Phys. Rev. C 95064326.

[10] N. Pillet, P. Quentin, J. Libert (2002) Nucl. Phys. A 697 141-163.

[11] H.N. Qasim, F.H. Al-Khudair (2020) Nucl. Phys. A 1002121962.

[12] S. Zhu (2020) Phys. Rev. C 102044326.

[13] A.M. Khalaf, A.M. Ismail, A.A. Zaki (2020) Nucl. Phys. A 996121704.

[14] V. Rani et al. (2021) Eur. Phys. J. A 57274. 
[15] T.J. Mertzimekis et al. (2016) NIM A 80756.

[16] I.E. Assimakis et al. (2018) arXiv:1811.01071.

[17] A. Martinou et al. (2021) Eur. Phys. J. A 5784.

[18] J. Wiederhold et al. (2019) Phys. Rev. C 99024316.

[19] A. Chalil, T.J. Mertzimekis, A. Zyriliou, P. Vasileiou, G. Zagoraios, A. Martinou, D. Bonatsos, N. Florea, C.R. Nita, C. Sotty, L. Stan, A. Turturica, N. Mărginean, (in preparation)

[20] D. Bucurescu, I. Căta-Danil, G. Ciocan, C. Costache, D. Deleanu, R. Dima, D. Filipescu, N. Florea, D.G. Ghiţă, T. Glodariu, M. Ivaşcu, R. Lică, N. Mărginean, R. Mărginean, C. Mihai, A. Negret, C.R. Niţă, A. Olăcel, S. Pascu, T. Sava, L. Stroe, A. Şerban, R. Şuvăilă, S. Toma, N.V. Zamfir, G. Căta-Danil, I. Gheorghe, I.O. Mitu, G. Suliman, C.A. Ur, T. Braunroth, A. Dewald, C. Fransen, A.M. Bruce, Zs. Podolyák, P.H. Regan, and O.J. Roberts (2016) NIM A 837 1-10.

[21] T. Beck, C. Costache, R. Lică, N. Mărginean, C. Mihai, R.E. Mihai, O. Papst, S. Pascu, N. Pietralla, C. Sotty, L. Stan, A.E. Turturică, V. Werner, J. Wiederhold, W. Witt (2020) NIM A 951163090.

[22] A. Dewald, O. Möller, P. Petkov (2012) Progress in Particle and Nuclear Physics 67 786-839. 